\title{
Postpartum Lactobacillus jensenii endocarditis in patient with bicuspid aortic valve
}

\author{
Anna Marciniak, PhD, Georgios T. Karapanagiotidis, MD, PhD, Mazin Sarsam, FRCS, and \\ Rajan Sharma, MD, London, United Kingdom
}

Lactobacillus is a rare cause of endocarditis and has been linked to structural heart disease, invasive procedures, prosthetic valves, and dental infections. We report for the first time a case of Lactobacillus endocarditis that occurred in a 31-year-old woman without a medical history 1 month after her first pregnancy and vaginal delivery involving not only the native aortic valve but also the posterior part of ascending aorta, the roof of the left atrium, and the intra-atrial septum, requiring a complex surgical procedure.

\section{CLINICAL SUMMARY}

A 31-year-old woman was admitted with a 1-month history of lethargy, fever, myalgia, and malaise since the delivery of her twins. This was her first pregnancy, and she delivered healthy babies by vaginal delivery. She was previously fit and well, with no medical problems.

On admission, the patient was febrile with a temperature of $39^{\circ} \mathrm{C}$ and a collapsing pulse, and on auscultation of the heart there was an early diastolic murmur. There were no cutaneous stigmata of endocarditis. Laboratory results demonstrated a hemoglobin level of $9.5 \mathrm{~g} / \mathrm{dL}$, white blood count of $22 \times 10^{9} / \mathrm{L}$, and C-reactive protein of $275 \mathrm{mg} / \mathrm{L}$. Urine dipstick was positive for blood $(+)$ and protein $(++)$, and 12-lead electrocardiogram showed sinus tachycardia with a PR interval of $226 \mathrm{~ms}$.

Transthoracic echocardiogram showed a bicuspid aortic valve with attached vegetation to both aortic cusps (Figure 1, A) and hyperdynamic left ventricular systolic function. Transesophageal echocardiogram confirmed a large vegetation attached to the aortic valve with severe aortic regurgitation (Figure 1,B) and revealed an aortic root abscess (Figure 1,C). Two sets of blood cultures, within 48 hours from the admission, grew Lactobacillus jensenii. The patient was allergic to penicillin, so intravenous teicoplanin ( $800 \mathrm{mg}$ once per day) and oral rifampicin (600 mg twice per day) were administered for the treatment

\footnotetext{
From the Department of Cardiology and Cardiothoracic Surgery, St George's Hospital, London, United Kingdom.

Disclosures: Authors have nothing to disclose with regard to commercial support.

Received for publication Nov 27, 2013; revisions received May 5, 2014; accepted for publication May 27, 2014; available ahead of print Aug 22, 2014.

Address for reprints: Georgios T. Karapanagiotidis, MD, PhD, St George's Hospital, Blackshaw Rd, London SW17 0QT, United Kingdom (E-mail: georgios. karapanagiotidis@nhs.net).

J Thorac Cardiovasc Surg 2014;148:e219-21

0022-5223/\$36.00

Copyright (c) 2014 by The American Association for Thoracic Surgery

http://dx.doi.org/10.1016/j.jtcvs.2014.05.096
}

of endocarditis. She underwent an aortic valve replacement with a mechanical prosthesis and aortic root repair after 4 weeks of antibiotic treatment. The total course of antibiotics lasted 10 weeks, including 4 weeks before and 6 weeks after the surgical treatment.

A sternotomy was performed, and cardiopulmonary bypass was established with arterial return to the ascending aorta and venous drainage with a separate superior vena cava and inferior vena cava cannula. The aortic valve was found to be bicuspid with fusion between the right and left cusps, and the vegetation was attached to both aortic cusps. The endocarditis involved the aortic-mitral continuity with extension onto the posterior wall of the aorta and the roof of the left atrium, and a communication across the upper part of the interatrial septum. The coronary arteries appeared normal. The aortic valve was excised, and the aorta was divided at the commissure between the left and the noncoronary cusp and into the abscess cavity localized at the base of the anterior leaflet of the mitral valve. The roof of the left atrium was also opened. All infected material was excised, including part of the wall of the aorta, the left atrium, and the interatrial septum. The interatrial septum was patched with a $1.5 \times 1.5-\mathrm{cm}$ circular bovine pericardium. Then, 2 diamond-shaped patches of bovine pericardium were used. The patches were sutured to the base of the anterior leaflet of the mitral valve up to the aortic-mitral continuity, and then they were separated: one to cover the roof of the left atrium and one to cover the posterior wall of the aorta (Figure 2, $A$ and $B$ ). A 25-mm mechanical St Jude valve (St Jude Medical Inc, St Paul, Minn) was inserted using interrupted 2-0 Ti-Cron sutures (Covidien, Dublin, Ireland). Pledgeted sutures were used where the valve was attached to the patch. The mitral valve leaflets were checked and found to have good motility and coaptation. The suturing of the patch over the roof of the left atrium and the posterior wall of the aorta was then completed. The rest of the aorta was closed directly with 5-0 Prolene sutures. The patient was transferred to the cardiac intensive care unit and extubated 6 hours after the operation. The postoperative course was uneventful, and on discharge, the echocardiogram showed a well-seated mechanical aortic valve prosthesis without paravalvular leakage.

\section{DISCUSSION}

Lactobacillus endocarditis is estimated to represent $0.05 \%$ to $0.4 \%$ of all endocarditis cases and was first described in $1938 .{ }^{1}$ Lactobacillus endocarditis has been 


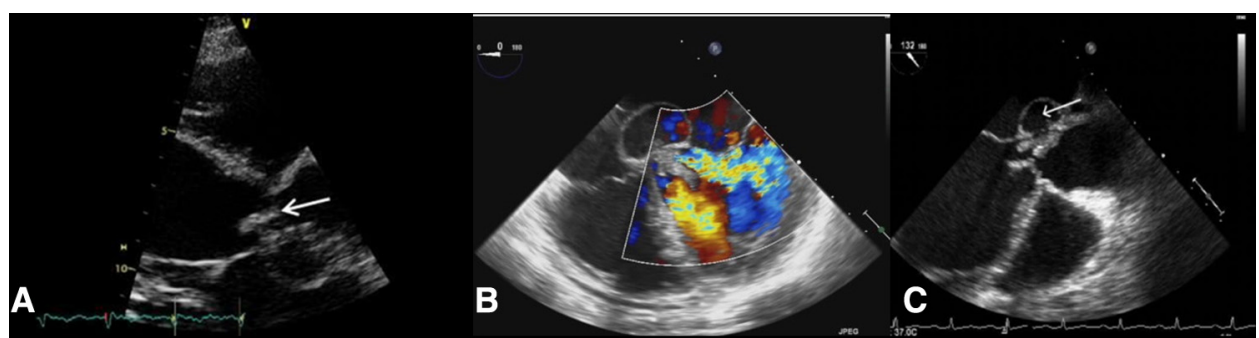

FIGURE 1. A, Transthoracic echocardiogram showing a vegetation attached to the aortic valve (white arrow indicates a vegetation). B, Transesophageal echocardiogram showing aortic regurgitation. C, Transesophageal echocardiogram showing an aortic root abscess (indicated by the arrow).

linked to structural heart disease, invasive procedures, prosthetic valves, and dental infections. ${ }^{2}$ Lactobacilli, including $L$ jensenii, are one of the most common of the normal bacterial flora of the female genital tract, which suggests that the vaginal flora was likely to be the main port of entry for the postdelivery endocarditis in the presented patient. The presence of a bicuspid aortic valve further increased the risk of infection.

This gram-positive rod has rarely been described in the literature. $^{2}$ There were only 5 case reports, and none of them had preexisting valvular disease. The previously described case of $L$ jensenii endocarditis after an induced abortion also supports the theory of transmission from the genital tracts. None of the reported cases were associated with bicuspid aortic valve or occurred after a normal delivery.

Surgical treatment of valve endocarditis was introduced in the early years of open surgery. However aortic valve endocarditis with associated periannular abscess still remains a surgically challenging disease with significantly increased mortality. ${ }^{3}$ Contemporary modes of surgery for periannular abscess/pseudoaneurysm involve drainage of the cavity, radical debridement of necrotic tissue, annular reconstruction of the destroyed annulus, and root replacement using an optimal conduit. Radical debridement is of primary importance and is the universally accepted procedure, which frequently requires annular reconstruction using a pericardial patch. ${ }^{4}$ This usually is followed by simple repair of defects or complex reconstructions of the left ventricular outflow tract and aortic root, involving pericardial or synthetic patches..$^{5}$ Despite the success obtained with these methods, there are studies reporting the superiority of autografts (Ross procedure) and homograft valve replacement for complex endocarditis., Homografts are not always available, and the Ross operation is complicated; therefore, they are not recommended in urgent situations. ${ }^{5}$ The reported case was an example of a complex endocarditis case involving the aortic valve with root abscess extending to the posterior part of the aorta, the roof of the left atrium, and the
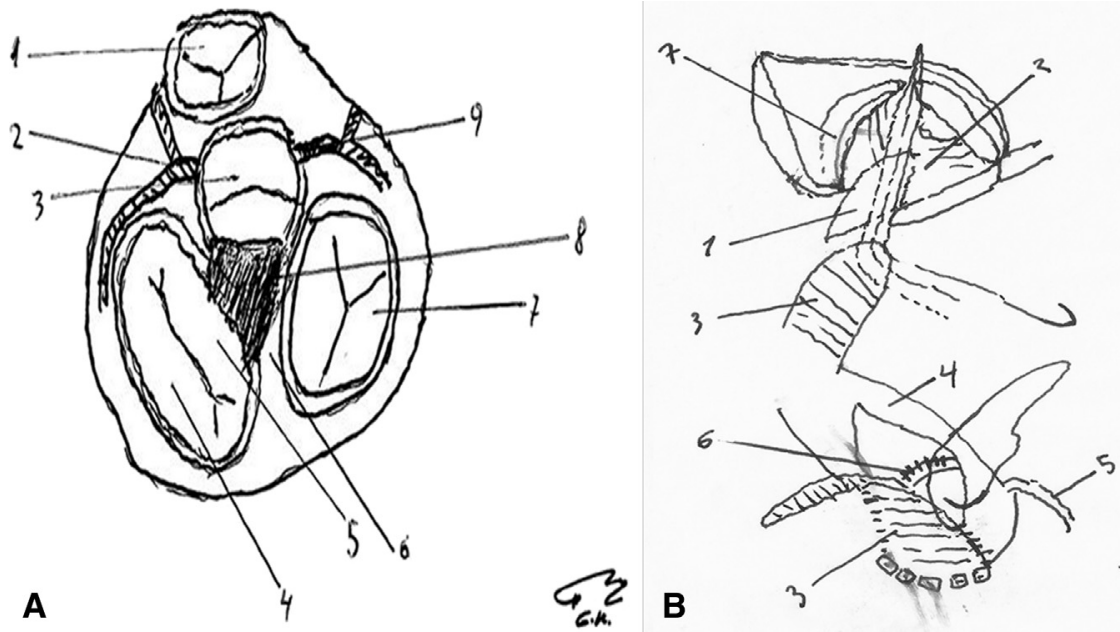

FIGURE 2. A, Short-axis view of the bovine pericardium patch between the anterior leaflet of the mitral valve and the aortic mitral continuity. 1 , Pulmonary valve. 2, Left coronary artery. 3, Bicuspid aortic valve. 4, Posterior leaflet of the mitral valve. 5, Anterior leaflet of the mitral valve. 6, Interatrial septum. 7, Tricuspid valve. 8, Bovine pericardium patch. 9, Right coronary artery. B, Long-axis view through open posterior wall of aortic root. 1, Left atrium through incision. 2, Anterior leaflet of mitral valve. 3, Bovine pericardium patch secured by pledgeted sutures. 4, Ascending aorta. 5, Right coronary artery. 6, Prosthetic valve annulus. 7, Aortic valve annulus. 
intra-atrial septum. This further required effective excision of the infected tissue. The interatrial septum was patched with bovine pericardium and the roof of the left atrium and the posterior wall of the aorta. The prosthetic valve replacement still remains the standard surgical approach despite the reported success with homografts, autografts, and xenografts. ${ }^{5}$

\section{CONCLUSIONS}

We report a rare case of aortic valve endocarditis caused by $L$ jensenii after a normal vaginal delivery in a patient with bicuspid aortic valve, which was complicated by severe aortic regurgitation and extensive aortic root abscess that further required a complex surgery.

\section{References}

1. Gasser F. Safety of lactic-acid bacteria and their occurrence in human clinical infections. Bull Inst Pasteur. 1994;92:45-67.

2. Brougui P, Raoult D. Endocarditis due to rare and fastidious bacteria Clin Microbiol Rev. 2001;14:177-207.

3. Knosalla C, Weng Y, Yankah AC, Siniawski H, Hofmeister J, Hammerschmidt R, et al. Surgical treatment of active infective aortic valve endocarditis with associated periannular abscess -11 year results. Eur Heart J. 2000;21:490-7.

4. Okada K, Okita Y. Surgical treatment for aortic periannular abscess/pseudoaneurysm caused by infective endocarditis. Gen Thorac Cardiovasc Surg. 2013;61:175-81.

5. Armani M, Schoevardts JC, Eucher P, Niicolas AL, Dion R, Kremer R. Extension of native aortic valve endocarditis: surgical considerations. Eur Heart J. 1995; 16(Suppl B):103-6.

\title{
Successful surgical treatment of rare Aspergillus terreus prosthetic valve endocarditis complicated by intracranial and mesenteric artery mycotic aneurysms
}

\author{
Rania A. R. Ahmad, MD, ${ }^{a}$ Syed T. Hussain, MD, ${ }^{b}$ Carmela D. Tan, MD, ${ }^{c}$ Gösta B. Pettersson, MD, PhD, ${ }^{b}$ \\ Daniel Clair, MD, ${ }^{\mathrm{d}}$ and Steven M. Gordon, MD, ${ }^{\mathrm{a}}$ Cleveland, Ohio
}

Aspergillus endocarditis (AE) is a rare and ominous cause of infective endocarditis. ${ }^{1-3} \mathrm{AE}$ is often disseminated and fatal, and detection of its source, establishment of the diagnosis, and treatment are highly challenging. We report an advanced case of prosthetic valve endocarditis caused by the species Aspergillus terreus, complicated by intracranial and superior mesenteric artery mycotic aneurysms, recent intracranial bleeding, and multiple peripheral embolic episodes.

\section{CLINICAL SUMMARY}

A 39-year-old man with a severely stenotic bicuspid aortic valve underwent mechanical aortic valve replacement in May 2013, complicated by complete heart block

\footnotetext{
From the Department of Infectious Disease, ${ }^{a}$ Medicine Institute; the Departments of Thoracic and Cardiovascular Surgery ${ }^{\mathrm{b}}$ and Vascular Surgery, ${ }^{\mathrm{d}}$ Heart and Vascular Institute; and the Department of Anatomic Pathology, ${ }^{\mathrm{c}}$ Laboratory Medicine Institute, Cleveland Clinic, Cleveland, Ohio.

R.A.R.A. is currently at Sheikh Khalifa Medical City, Abu Dhabi, United Arab Emirates. Disclosures: Authors have nothing to disclose with regard to commercial support.

Received for publication June 4, 2014; accepted for publication June 5, 2014; available ahead of print Aug 23, 2014.

Address for reprints: Gösta B. Pettersson, MD, PhD, Department of Thoracic and Cardiovascular Surgery, Cleveland Clinic, 9500 Euclid Ave, Desk J4-1, Cleveland, OH 44195 (E-mail: petterg@ccf.org).

J Thorac Cardiovasc Surg 2014;148:e221-3

$0022-5223 / \$ 36.00$

Copyright (C) 2014 by The American Association for Thoracic Surgery

http://dx.doi.org/10.1016/j.jtcvs.2014.06.084
}

requiring permanent pacemaker implantation. Four months later, he presented with lower extremity embolus requiring embolectomy. The removed embolus grew Aspergillus, not further speciated. Echocardiography showed vegetations at the sinotubular junction and in the aortic root, and the patient underwent reoperation with removal of infectious vegetations and augmentation of the aorta with bovine pericardial patch, without replacement of the valve prosthesis. The vegetations grew Aspergillus species. During treatment with amphotericin B and voriconazole, the patient had developed an abscess in the left lower forearm requiring surgical drainage. The culture grew Aspergillus, prompting transfer to our institution in February 2014.

Within 24 hours of arrival, the patient had acute embolic occlusion of the right superficial femoral and profunda femoris arteries requiring urgent thromboembolectomy. The embolus grew A terreus, speciated for the first time, and the antifungal treatment was changed from amphotericin $B$ to voriconazole and micafungin sodium (INN micafungin) on the basis of sensitivity. Echocardiography showed large, mobile vegetations in the proximal aorta near the sinotubular junction (Figure 1, A). Computed tomographic scan of the abdomen revealed a 6-cm superior mesenteric artery aneurysm with surrounding inflammation (Figure 1, $B)$. Head computed tomography showed multiple small 